Keywords: sorafenib; multikinase inhibitor; advanced hepatocellular cancer; primary care trusts; health-care rationing; supportive care

\title{
Sorafenib for advanced hepatocellular carcinoma (HCC): impact of rationing in the United Kingdom
}

\author{
D H Palmer ${ }^{\star, 1,2,4}$, S A Hussain *,1,2,4 , A J Smith ${ }^{2}$, S Hargreaves ${ }^{3}$, Y T Ma ${ }^{2}$, D Hull ${ }^{2}$, P J Johnson ${ }^{1,2}$ and P J Ross ${ }^{3}$ \\ ${ }^{1}$ Cancer Research UK Centre, University of Liverpool, Daulby Street, Liverpool L69 3GA, UK; ${ }^{2}$ Cancer Research UK, School of \\ Cancer Sciences, University of Birmingham, Vincent Drive, Edgbaston, Birmingham B15 2TT, UK and ${ }^{3}$ King's College Hospital and \\ Guy's and St Thomas' NHS Foundation Trusts, London, UK
}

Background: The prognosis for hepatocellular carcinoma (HCC) is dependent upon tumour stage, performance status (PS), severity of underlying liver disease, and the availability of appropriate therapies. The unavailability of sorafenib may have a significantly adverse effect on the prognosis of UK patients with advanced HCC. During the study period, access to sorafenib was at the discretion of local health funding bodies, a process that may delay or deny access to the drug and that remains in place for Wales, Scotland, and Northern Ireland. Here, we attempt to address the impact of this system on patients with advanced HCC in the United Kingdom.

Methods: This is a retrospective study performed in the two largest specialist hepatobiliary oncology units in the United Kingdom. Funding applications were made to local funding bodies for patients with advanced HCC for whom sorafenib was considered appropriate (advanced HCC not suitable for loco-regional therapies, compensated chronic liver disease, PS 0-2).

Results: A total of 133 applications were made, of which 57 (43\%) were approved and 76 (57\%) declined. Demographics and prognostic factors were balanced between the two groups. This cohort had a number of adverse prognostic features: patients were predominantly PS 1-2; the majority had multifocal disease with the largest lesion being $>5 \mathrm{~cm}$; and macroscopic vascular invasion, metastases, and AFP $>1000 \mathrm{ng} \mathrm{ml}^{-1}$, were each present in one-third of cases. The median time from application to funding decision was 17 days (range 3-260 days). For the primary 'intention-to-treat' analysis, median overall survival was 4.1 months when funding was declined, and 9.5 months when funding was approved (hazard ratio (HR) 0.48; 95\% Cl 0.3186-0.7267; $P=0.0005)$.

Conclusion: These data support the use of sorafenib for patients with advanced HCC as an effective intervention. In the United Kingdom, this applies to a relatively small group of patients, estimated to total $\sim 800$ per year who, unfortunately, do not survive long enough to themselves lobby for the availability of this drug. These data provide a comparison of sorafenib with supportive care and demonstrate the potential detrimental impact on patient outcomes of rationing health-care resources on the basis of cost.

With an estimated 700000 new cases per year, hepatocellular carcinoma (HCC) represents the fifth commonest cancer and is the third leading cause of cancer death worldwide. Although cases occur predominantly in the Far East and Sub-Saharan Africa, the incidence is rising in the West, including the United Kingdom, largely due to an increasing incidence of Hepatitis C virus infection, alcoholic liver disease, and non-alcoholic steatohepatitis related to obesity and type II diabetes (El-Serag and Mason, 1999).

*Correspondence: Professor DH Palmer, E-mail: daniel.palmer@liverpool.ac.uk or Dr SA Hussain; E-mail: syed.hussain@liverpool.ac.uk
${ }^{4}$ DHP and SAH contributed equally to this work

Received 11 April 2013; revised 10 June 2013; accepted 1 July 2013; published online 23 July 2013

(c) 2013 Cancer Research UK. All rights reserved 0007-0920/13 
The majority of patients present with disease that is too advanced for curative surgery by either resection or transplantation. Randomised phase III placebo-controlled trials have demonstrated a survival advantage for sorafenib, a small molecule multikinase inhibitor of the mitogen-activated protein kinase pathway and of vascular endothelial growth factor receptors, compared to best supportive care in patients with advanced HCC (Llovet JM et al, 2008; Cheng et al, 2009). On this basis, sorafenib has been accepted as the standard of care in this context. However, in the current health economic climate, the potential financial burden of novel anticancer drugs is of increasing relevance. In England and Wales, the availability of new drugs is determined by the National Institute for Health and Clinical Excellence (NICE) and in Scotland by the Scottish Medicines Consortium (SMC) based on an assessment of clinical- and cost-effectiveness. Following negative NICE and SMC appraisals, sorafenib was not routinely available within the National Health Service (NHS) in the United Kingdom.

Since the prognosis for HCC is dependent upon tumour stage, performance status (PS), severity of underlying liver disease, and the availability of appropriate therapies, the unavailability of sorafenib may have a significantly adverse effect on the prognosis of UK patients with advanced HCC (Palmer and Johnson, 2006).

During the study period, access to sorafenib was at the discretion of local health funding bodies through individual funding requests, a process that may delay or deny access to the drug, with decisions being made on a case-by-case basis. Here, we attempt to address the impact of this system on patients with advanced HCC in the United Kingdom.

\section{PATIENTS AND METHODS}

This is a multicentre retrospective study performed at the two largest specialist hepatobiliary oncology units of the United Kingdom (Kings College Hospital, London, and Queen Elizabeth Hospital, Birmingham). Institutional approvals for this study were obtained. Between July 2007 and May 2009, funding applications were made to local funding bodies for patients with advanced HCC for whom sorafenib was considered appropriate (advanced HCC not suitable for loco-regional therapies, compensated chronic liver disease and PS 0-2). The primary outcome measure was overall survival from the date of application. The secondary outcome measure was overall survival in those receiving at least one dose of sorafenib.

\section{RESULTS}

A total of 133 applications were made to 42 local funding bodies in England and Wales, of which 57 (43\%) were approved and 76 (57\%) declined. The criteria for application were uniform across both Centres and comprised clinical information to indicate that, in the treating clinician's opinion, sorafenib was the most appropriate therapy - that is, it had a good PS (WHO PS 0-2); well-compensated background chronic liver disease; not a suitable candidate for loco-regional therapies (surgery, transplantation, local ablation, and TACE). On this basis, decisions on whether to fund were not apparently based on clinical variables. Indeed, as subgroup analyses of the sorafenib-randomised trials indicate similar benefit across all subgroups, there are no known predictive variables that the funding bodies could have used to select patients more likely to benefit from treatment. This is reflected in the key demographic and prognostic factors, which are summarised in Table 1. In general, these were balanced between the two groups and between the two centres, and statistical comparison of each variable revealed no significant differences. Notably, this cohort had a number of adverse prognostic features: patients were predominantly PS 1-2; the majority had multifocal disease with the largest lesion being $>5 \mathrm{~cm}$; and macroscopic vascular invasion, metastases, and AFP $>1000 \mathrm{ng} \mathrm{ml}^{-1}$ were each present in approximately one-third of cases. The median time from application to funding decision was 17 days (range 3-260 days). For the primary 'intention-to-treat' analysis, the median overall survival was 4.1 months when funding was declined, and 9.5 months when funding was approved (hazard ratio (HR) 0.48; 95\% CI $0.3186-0.7267 ; P=0.0005$; Figure 1). In 14 of the 57 cases where funding was approved, in the time awaiting the funding body decision, the clinical condition deteriorated such that treatment could not be commenced. The median survival for the 43 patients who received at least one dose of sorafenib was 10.7 months (HR 0.38; 95\% CI 0.25-0.59; $P<0.0001$ ). In those receiving sorafenib, the median duration of treatment was 5.1 months. There was a higher proportion of patients with metastatic disease in the unfunded group, which conceivably could negatively influence the survival in this group. However, a sensitivity analysis

\begin{tabular}{|c|c|c|c|}
\hline Demographic & $\begin{array}{c}\text { All } \\
\text { patients }\end{array}$ & $\begin{array}{c}\text { Sorafenib } \\
\text { funded }\end{array}$ & $\begin{array}{l}\text { Sorafenib } \\
\text { not funded }\end{array}$ \\
\hline Patient number & 133 & 57 & 76 \\
\hline Kings patients & 71 & 30 & 41 \\
\hline Birmingham patients & 62 & 27 & 35 \\
\hline$M: F$ & $108: 25$ & $52: 5$ & $56: 20$ \\
\hline Median age (range) & $62(16-86)$ & $61(16-82)$ & $62(17-86)$ \\
\hline PS $0: 1: 2(\%)$ & $19: 49: 32$ & $20: 48: 32$ & $18: 48: 34$ \\
\hline Child-Pugh A & $82 \%$ & $84 \%$ & $80 \%$ \\
\hline$A F P \geqslant 1000$ & $31 \%$ & $30 \%$ & $31 \%$ \\
\hline Multifocal & $70 \%$ & $65 \%$ & $75 \%$ \\
\hline Largest lesion $>5 \mathrm{~cm}$ & $68 \%$ & $78 \%$ & $60 \%$ \\
\hline Macroscopic vascular invasion & $34 \%$ & $41 \%$ & $29 \%$ \\
\hline Extrahepatic metastases & $39 \%$ & $30 \%$ & $46 \%$ \\
\hline
\end{tabular}

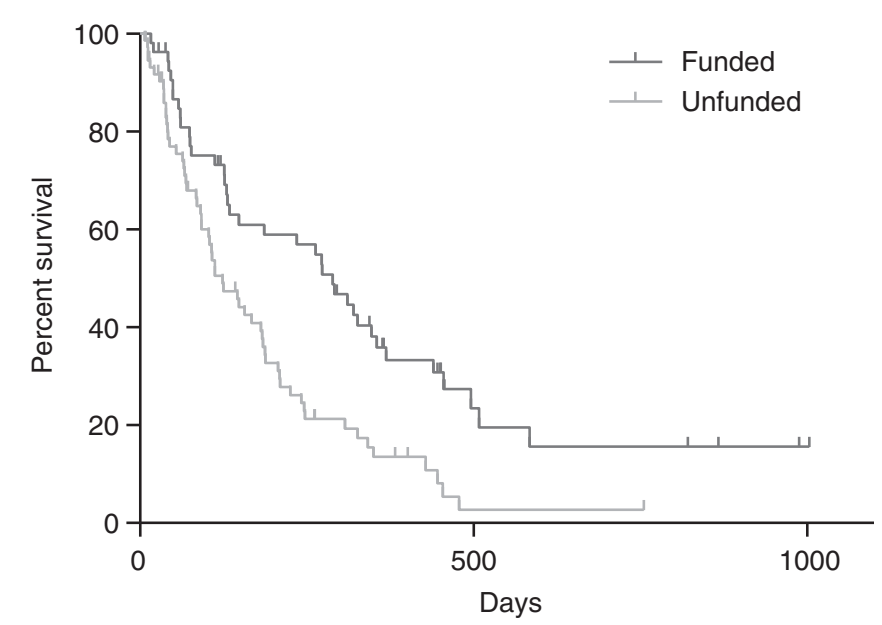

Figure 1. Survival proportions in patients for whom an application for sorafenib funding was made. 
excluding all patients with metastases did not significantly affect the data, again indicating that differences in survival are likely due to treatment effects rather than due to imbalances in prognostic variables between the two groups (non-metastatic patients, median survival funded vs unfunded: 8.95 vs 3.7 months; HR 0.51, 95\% CI $0.32-0.82 ; P=0.0061)$.

Four patients in this series had fibrolamellar variant HCC (three funding-approved, one declined). It is considered that this variant may confer a better prognosis than classical HCC, which may, at least in part, be due to the younger age at presentation (median 20-30 years) and the absence of underlying cirrhosis. These factors may permit more radical surgical treatments at initial presentation, although relapse with metastatic disease is common. Upon relapse, a variety of treatments including further surgery and conventional chemotherapy may be employed such that, by the time sorafenib is considered, prognosis is likely to be poor. Taking into account a potential confounding influence of fibrolamellar patients, a further survival analysis excluding these patients demonstrated the median overall survival of 8.98 and 3.68 months (HR 0.45; 95\% CI 0.29-0.69; $P=0.0002$ ) in funded and unfunded cases, respectively, indicating no confounding influence by these patients.

\section{DISCUSSION}

Sorafenib is the first systemic agent to demonstrate a statistically significant survival benefit in adequately powered, randomised phase III trials for patients with advanced HCC and is considered the standard of care in this context. Although these trials represented patients from Europe and the Far East, our study suggests a similar benefit for patients in the UK. Notably, these patients had a number of adverse prognostic features compared with those recruited to the phase III trials, including poorer PS and greater tumour burden. Despite this, the survival advantage for these patients compares favourably to the randomised trials. Of note, the HR reported here $(0.48)$ is somewhat better than that reported in the SHARP trial (0.69) (Llovet et al, 2008). This may, in part, be due to the relatively worse outcome for untreated patients reflecting a number of adverse prognostic variables present in this study population and additionally may reflect the experience of two high-volume liver units and the evolution of experience in managing toxicities and maintaining dose intensity for sorafenibtreated patients since the original publication of the trial data. Although treatment in this study was not randomly assigned, the funding applications were made using the same criteria for suitability for sorafenib, and the baseline demographics between the two groups were generally balanced, suggesting that the improved outcome was due to a treatment effect rather than due to confounding prognostic variables.

The SMC and NICE single technology appraisals of sorafenib for advanced HCC recommended that the drug should not be available on the basis of cost effectiveness (National Institute for Health and Clinical Excellence and Scottish Medicines Consortium websites). This decision was based on a calculated cost per incremental quality-adjusted life-year (QALY) gain of approximately $£ 64000$, which exceeds the standard threshold of approximately $£ 30000$ for drugs used to prolong survival near the end of life. This calculation was derived using the HR for overall survival of 0.69 reported in the phase III trials. However, recalculation of this figure using the $\mathrm{HR}$ of 0.48 reported here reduces the cost per incremental QALY to approximately $£ 30000$. The data reported here support the use of sorafenib for patients with advanced HCC as clinical and cost-effective interventions. In the United Kingdom, this applies to a relatively small group of patients, estimated to total $\sim 800$ per year who, unfortunately, do not survive long enough to themselves lobby for the availability of this drug.

Our results provide a comparison of sorafenib with supportive care and demonstrate the potential detrimental impact on patient outcomes of rationing health-care resources on the basis of cost.

\section{CONFLICT OF INTEREST}

DHP, PJJ, and SAH have received unrestricted educational grants from Bayer Schering Pharma. The remaining authors declare no conflict of interest.

\section{REFERENCES}

Cheng AL, Kang YK, Chen Z, Tsao CJ, Qin S, Kim JS, Luo R, Feng J, Ye S, Yang TS, Xu J, Sun Y, Liang H, Liu J, Wang J, Tak WY, Pan H, Burock K, Zou J, Voliotis D, Guan Z. Efficacy and safety of sorafenib in patients in the Asia-Pacific region with advanced hepatocellular carcinoma: a phase III randomised, double-blind, placebo-controlled trial. Lancet Oncol (2009) 10(1): 25-34.

El-Serag HB, Mason AC. Rising incidence of hepatocellular carcinoma in the United States. N Engl J Med (1999) 340(10): 745-750.

Llovet JM, Ricci S, Mazzaferro V, Hilgard P, Gane E, Blanc JF, de Oliveira AC, Santoro A, Raoul JL, Forner A, Schwartz M, Porta C, Zeuzem S, Bolondi L, Greten TF, Galle PR, Seitz JF, Borbath I, Häussinger D, Giannaris T, Shan M, Moscovici M, Voliotis D, Bruix J. SHARP Investigators Study Group. Sorafenib in advanced hepatocellular carcinoma. N Engl J Med (2008) 359(4): 378-390.

National Institute for Health and Clinical Excellence. Final Appraisal Determination. Sorafenib for the treatment of advanced hepatocellular carcinoma http://www.nice.org.uk/nicemedia/live/12019/46312/46312.pdf.

Palmer DH, Johnson PJ (2006) Prognostic factors in hepatocellular carcinoma. In: UICC Prognostic Factors in Cancer. 3rd edn. John Wiley \& Sons: Hoboken, NJ, USA.

Scottish medicines consortium. website http://www.scottishmedicines.org.uk/ files/sorafenib_Nexavar__FINAL_JUNE_2008_for_website.pdf.

This work is published under the standard license to publish agreement. After 12 months the work will become freely available and the license terms will switch to a Creative Commons AttributionNonCommercial-Share Alike 3.0 Unported License. 